\title{
Gene flow from the gorilla lineage in the Late Miocene as the "missing link"
}

ABSTRACT: The "missing link" as a concept comes from that there is no continuous evolutionary model for the origin of the human species. There is now conclusive evidence that the speciation of the Homo lineage was caused by the common ancestor of humans and chimpanzees interbreeding with the gorilla lineage around 6 million years ago, an event that also led to the speciation of the Paranthropus lineage. The hybridization of two separate lineages explains the absence of a single continuous lineage. Evidence for an interbreeding event in the Late Miocene can be seen as introgression of around $30 \%$ of the gorilla genome into the Pan-Homo clade, with lineage sorting between Pan and Homo where $15 \%$ of the gorilla genome is closer to humans, and another $15 \%$ being closer to the chimpanzee lineage. (Scally, 2012) The interbreeding event can also be read in the genomes of both Gorilla, Pan and Homo as fragments of mitochondrial DNA that during the event 6 million years ago was inserted into the genome as a NUMT ("nuclear mitochondrial DNA segment"), a pseudo-gene, that originates from the gorilla lineage (Popadin, 2017) and has been transferred to all three lineages.

\section{The introgression speciation model and a revised phylogenetic tree}

The NUMT data (Popadin, 2017), and the lineage sorting data (Scally, 2012) shows that the gorilla lineage and the common ancestor of humans and chimpanzees interbred 6 million years ago, causing the speciation of the lineage that led to both Australopithecus and Paranthropus. The introgression speciation model predicts that the Paranthropus lineage split from the Australopithecus lineage as early as 5-6 million years ago.

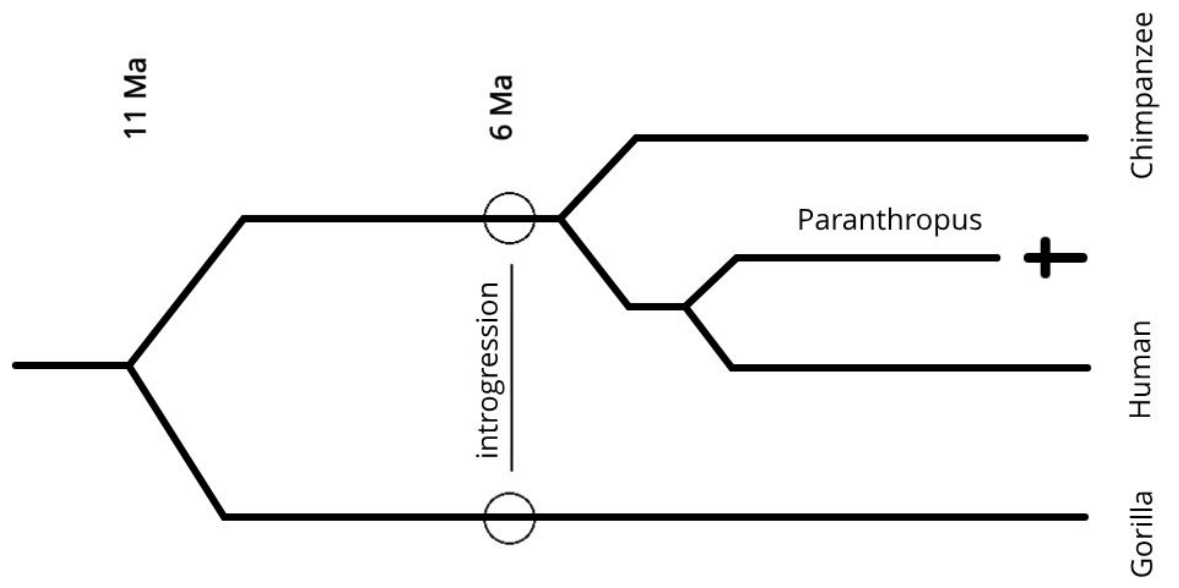

Fig 1. Revised phylogenetic tree showing how introgression caused the speciation of Homo. 


\section{The Pliocene hominin diversity is explained by interbreeding between lineages separated by $\mathbf{4 . 5}$ million years}

The introgression speciation model predicts the Pliocene hominin diversity (Haile-Selassie, 2015, 2016; Wood, 2016) that has been increasingly evident from the fossil record, with both the Australopithecus and Paranthropus lineages dating back to a common ancestor that speciated as a result of the interbreeding.

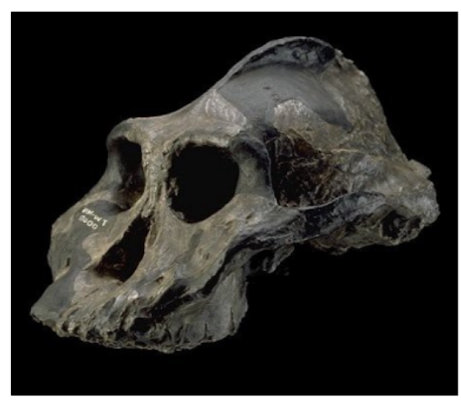

Paranthropus aethiopicus 2.8-2.3 Ma

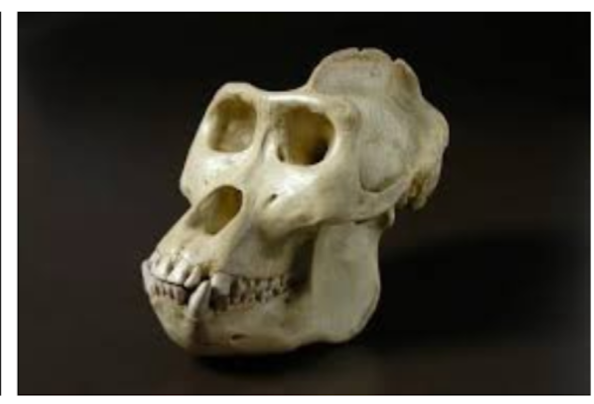

Gorilla gorilla

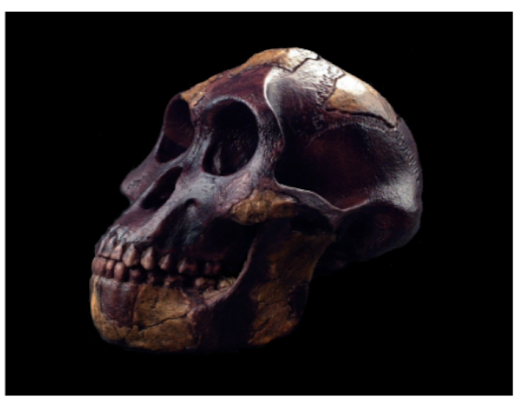

Australopithecus afarensis 3.7-2.9 Ma

Fig 2. Introgression from Gorilla caused the speciation of both Australopithecus and Paranthropus.

\section{Paranthropus deyiremeda and the Burtele foot (BRT-VP-2/73)}

The discovery of fossil remnants of Paranthropus deyiremeda (meaning "close relative") in 2011 (classified as $A u$. deyiremeda in 2015 by paleoanthropologist Yohannes Haile-Selassie) from 3.3-3.5 Ma was to be expected from the introgression speciation model, and the discovery of 3.3-3.5 million year old foot with an opposable great toe, BRT-VP-2/73, also at Woranso-Mille, fits with the introgression speciation model, the Australopithecus and Paranthropus lineages adapted to separate niches.

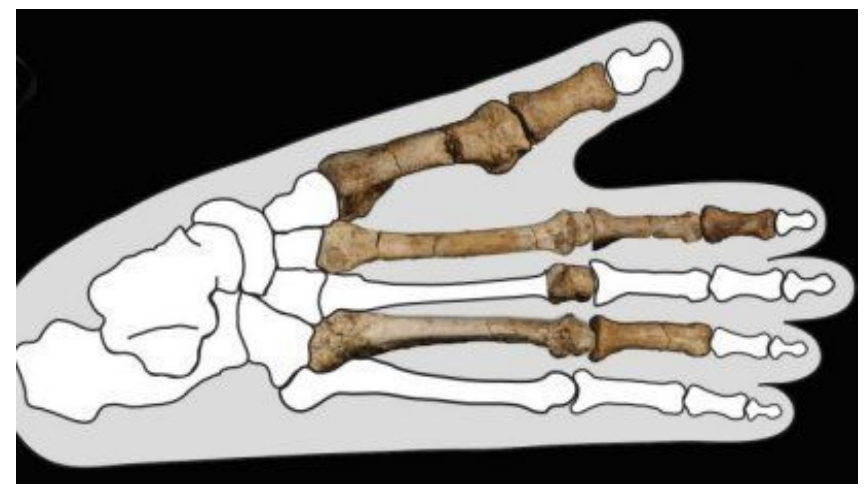

Fig 3. Fossil record of the introgression speciation model seen in Paranthropus deyiremeda. 


\section{The NUMT on chromosome 5 as conclusive evidence of an introgression speciation model}

Mitochondria is inherited separately from nuclear DNA, and fragments of mitochondrial DNA are known to get inserted into nuclear DNA to form NUMTs, i.e. nuclear pseudogenes of the mtDNA. There is a NUMT sequence on chromosome 5 shared by gorillas, chimpanzees, and humans, shown from mutation rates to date back to 6 million years ago, a result of interbreeding between lineages that had diverged as much as $\sim 4.5 \mathrm{Myr}$ prior to the interbreeding event. (Popadin, 2017) The NUMT on chromosome 5 shares affinities with the gorilla lineage mtDNA (Popadin, 2017) suggesting that it originates from the gorilla lineage.

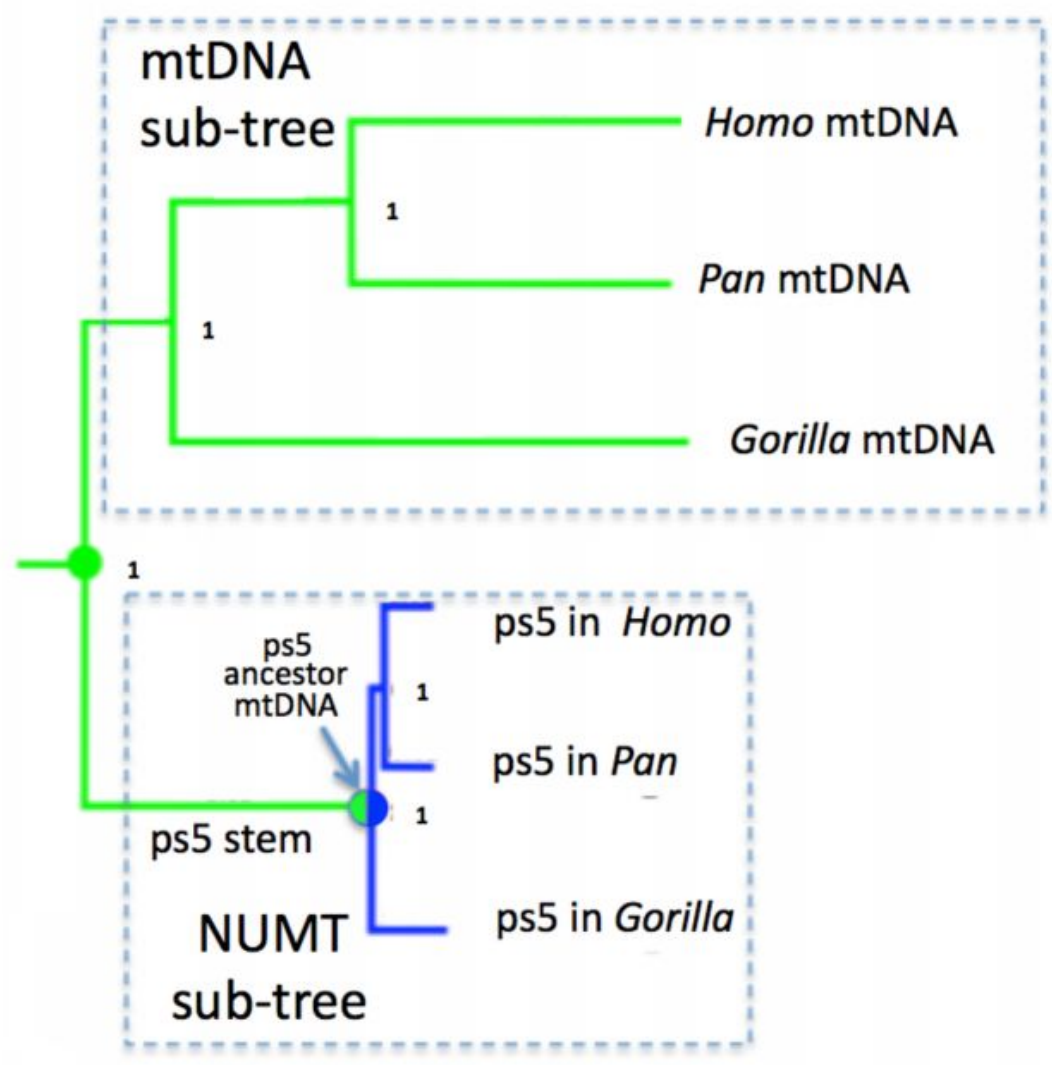

Fig 4. NUMT on chromosome 5 shared by Gorilla, Pan and Homo, that had diverged 4.5Myr prior to the introgression event, $11 \mathrm{Ma}$ when the Gorilla lineage split from the common ancestor of Pan and Homo. Image from Popadin et al, 2017. 


\section{Lineage sorting in humans and chimpanzees from introgression of genes}

Scally et al showed that $30 \%$ of the gorilla genome exhibits lineage sorting with the human genome and chimpanzee genome (Scally, 2012), with 15\% of the gorilla genome being closer to human than to chimpanzee, and vice versa, that the amount of lineage sorting we see where gorillas and humans are closest together is the same as the amount where gorillas and chimpanzees are closest to each other, a result of gene transfer from the interbreeding event 6 million years ago that also transferred the NUMT on chromosome 5 (Popadin, 2017) to all three lineages.

\section{The gorilla as the "missing link" and the Pthirus pubis host switch 3.3 $\mathrm{Ma}$}

The evolutionary history of anthropoid primate lice includes a host switch from the gorilla lineage to Homo around 3.3 million years ago (Reed, 2007).

\section{Host Switch}

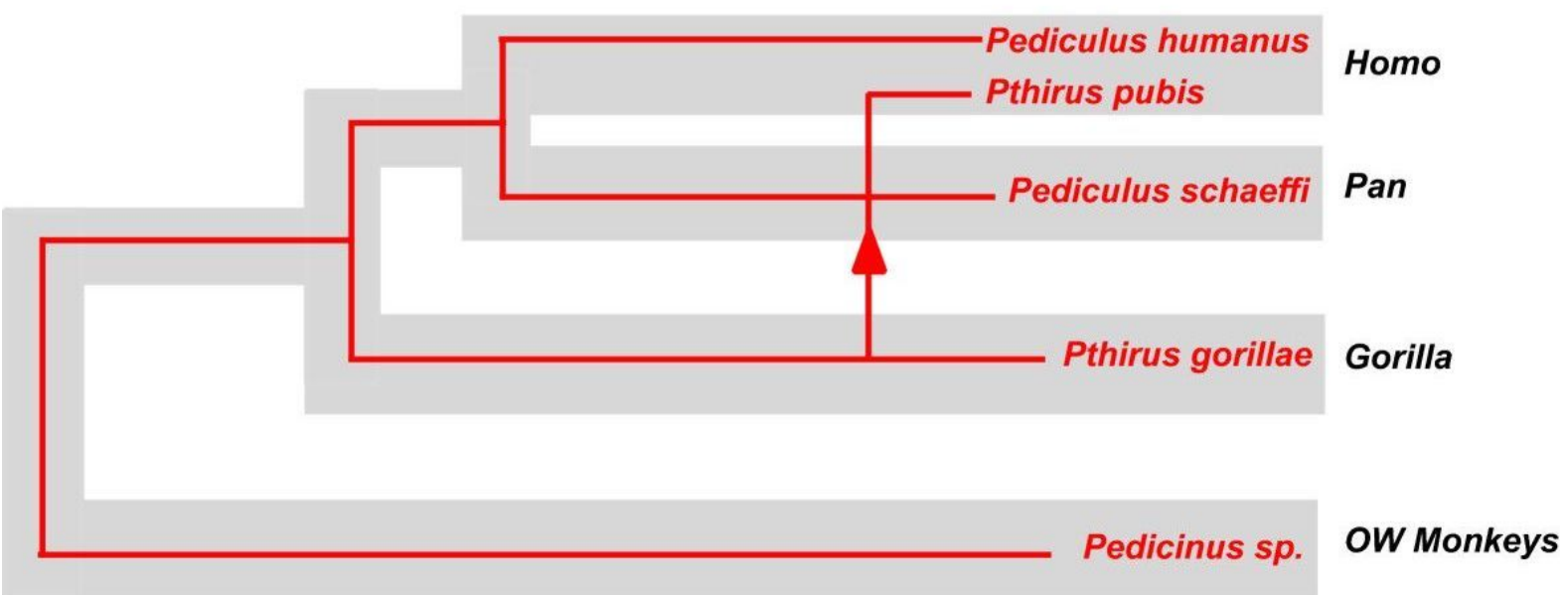

Fig 5. Reconstruction showing perfect cospeciation between with hosts and parasites with the exception of a single host switch of Pthirus sp. from gorillas to humans (marked by an arrow). Image from Reed et al, 2007. 


\section{References}

Haile-Selassie, Y., Gibert, L., Melillo, S. M., Ryan, T. M., Alene, M., Deino, A., ... Saylor, B. Z. (2015). New species from Ethiopia further expands Middle Pliocene hominin diversity. Nature, 521(7553), 483-488.

https://doi.org/10.1038/nature14448

Haile-Selassie, Y., Melillo, S. M., \& Su, D. F. (2016). The Pliocene hominin diversity conundrum: Do more fossils mean less clarity? Proceedings of the National Academy of Sciences, 113(23), 6364-6371.

https://doi.org/10.1073/pnas.1521266113

Popadin, K., Gunbin, K., Peshkin, L., Annis, S., Fleischmann, Z., Kraytsberg, G., ... Khrapko, K. (2017, May 9). Mitochondrial pseudogenes suggest repeated inter-species hybridization in hominid evolution. Cold Spring Harbor Laboratory. https://doi.org/10.1101/134502

Reed, D. L., Light, J. E., Allen, J. M., \& Kirchman, J. J. (2007). Pair of lice lost or parasites regained: the evolutionary history of anthropoid primate lice. BMC Biology, 5(1), 7. https://doi.org/10.1186/1741-7007-5-7

Scally, A., Dutheil, J. Y., Hillier, L. W., Jordan, G. E., Goodhead, I., Herrero, J., .. Durbin, R. (2012). Insights into hominid evolution from the gorilla genome sequence. Nature, 483(7388), 169-175.

$\underline{\text { https://doi.org/10.1038/nature10842 }}$

Wood, B., \& K. Boyle, E. (2016). Hominin taxic diversity: Fact or fantasy? American Journal of Physical Anthropology, 159, 37-78. https://doi.org/10.1002/ajpa.22902 